\title{
Muscle Injuries: 2020 Update of the I.S.Mu.L.T. Classification
}

\author{
G. Nanni ${ }^{1}$, A. Frizzieroํ, R. Di Miceli ${ }^{1}$, F. Vittadini ${ }^{3}$, P. Finotti ${ }^{4}$, J. Gamberini ${ }^{1}$, F. Oliva ${ }^{5}$, \\ M. Freschi ${ }^{6}$, M. Buckthorpe ${ }^{1,7}$, N. Maffulli8-10 \\ ${ }^{1}$ Department of Education and Research, Isokinetic Medical Group, FIFA Medical Centre of Excellence, Bologna, \\ Italy \\ 2 Department of Medicine and Surgery, University of Parma, Parma, Italy \\ ${ }^{3}$ Department of Physical and Rehabilitation Medicine, Casa di Cura Policlinico S. Marco, Venice, Italy \\ ${ }^{4}$ Department of Physical and Rehabilitation Medicine, University of Padova, Padova, Italy \\ ${ }^{5}$ Department of Musculoskeletal Disorders, School of Medicine and Surgery, University of Salerno, Italy \\ ${ }^{6}$ Isokinetic Medical Group, AC Juventus Turin Italy \\ 7 Faculty of Sport, Health and Applied Science, St Mary's University, Twickenham, London, UK \\ ${ }^{8}$ Department of Musculoskeletal Disorders, School of Medicine and Surgery, University of Salerno, Fisciano, Italy \\ 9 Orthopedic Clinic, San Giovanni di Dio e Ruggi D'Aragona Hospital, Hospital of Salerno, Salerno, Italy \\ ${ }^{10}$ Barts and the London School of Medicine and Dentistry, Centre for Sports and Exercise Medicine, Queen Mary \\ University of London, London, England
}

\section{CORRESPONDING AUTHOR:}

Antonio Frizziero

Department of Medicine and Surgery

University of Parma

via A. Gramsci 14

Parma, Italy

E-mail: antonio.frizziero@unipr.it

DOI:

10.32098/mltj.04.2020.03

LEVEL OF EVIDENCE: 5

\section{SUMMARY}

It is becoming increasingly important to have a common terminology, as international and shared as possible, beginning with the classification of injuries. This can then aid in correct diagnosis, a focus prognosis, and appropriate therapy. The most adopted classification system within practice must thus be both accurate and complete but also clinically useful for those less familiar in injury management.

Muscle injuries are divided into two main categories according to the mechanism of onset: direct muscle injuries caused by the impact of a blunt body against the muscular belly and in indirect muscle injuries, caused by a phenomenon of overstretching or eccentric contraction causing internal disruption on the muscle-tendon unit.

For the clinician who faces these pathologies, it is essential to utilize a classification system which considers both the clinical findings and information from diagnostic investigations, such as ultrasound and MRI. The multi-modal approach to muscle injury assessment (clinical, ultrasound and possibly resonance imaging) seems essential for a correct clinical framework and the assessment of the severity of injury, to subsequently determine the most appropriate therapeutic pathway. In recent years, numerous classifications have been published, to attempt to arrive at a standardized common language, but in most cases it has failed to make all the professional figures involved in the dialogue on rehabilitation. For an appropriate classification system, it is important to adopt correct terminology and avoid terms such as 'elongation' or 'strain'.

The objective of this paper is not to deeply address the diagnostic and therapeutic approach of muscle injuries, but to renew the standard scientific language related to muscle injuries among the various professional figures involved in rehabilitation starting from the already shared I.S.Mu.L.T. 2014 muscle injuries classification (1).

\section{KEY WORDS}

Muscle injury; classification; sport injury; muscle trauma; prognosis. 


\section{INTRODUCTION}

Muscle injuries are common, particularly during activities that involve high speed running, throwing and jumping $(1,2)$. Despite a wide variability between the different sports, the literature data report for them an overall amount of $10-55 \%$ of all acute injuries in athletes (3). These injuries frequently involve the lower limbs, particularly the hamstrings, quadriceps (rectus femoris) and gastrocnemius (medial head) muscle groups (4).

Recent scientific literature have demonstrated that the risk of a muscle injury is the result of complex interactions between different factors (5). Risk factors can be divided into intrinsic (related to athlete's characteristics) and extrinsic ones (related to the external environment) and they can be distinguished as modifiable and unmodifiable factors. The aim of injury prevention programs is to reduce or eliminate modifiable risk factors and control or over-compensate for non-modifiable risk factors (6). Age is an important predisposing factor for muscle injuries as a consequence of age related changes within the muscle, such as the re-arrangement of motor units and the denervation processes (7). A positive anamnesis for previous muscle injuries is related to a higher risk for other muscle lesions, both in the same muscular group and in other districts, because of the development of fibrotic tissues and/or acquired biomechanical alterations (8). Reduced muscle flexibility, joint range of motion and muscle strength are considered as modifiable risk factors, although the evidence is equivocal (9). Also, psycho-social factors (e.g. stressful events) appear to play a significant role for injuries (10). Higher levels of competition, lower recovery time between matches, higher intensity and overall volume of training time are associated with increased risk for muscle lesions (11). Match play carriers a much higher risk for muscle injury than training, for both adult professional and young players (12).

The diagnosis of a muscle injury is usually based on anamnestic data and clinical examination, supported by diagnostic imaging (13). The anamnesis focuses on the type of pain perceived, its localization and onset, the traumatic mechanism and the timing of the match or training. Furthermore, clinicians should investigate about history of previous muscle injuries of the same muscle group or adjacent joint muscles. Clinical examination is based on inspection, palpation, assessment of the range of motion, stretching and functional tests (14).

Diagnostic imaging is very important, not only for the early diagnosis and classification of the injury, but also to check the healing status, any complications during the treatment process, and to support prediction of return to play (RTP) times (15-16). Magnetic resonance imaging (MRI) is indicated when clinical tests and ultrasound information conflict (especially in professional athletes or in the case of muscles difficult to study in ultrasound such as psoas, obturators), as well as in the case of suspected subtotal or complete muscle injury, tendon involvement or bone-tendon unit avulsions (17).

Within the literature, there are a few classification systems which have been scientifically validated based on research demonstrating an association between the muscle injury grade classification and RTP times $(18,19)$. In order to promote an international terminology which could classify muscle injuries and aid in correct injury diagnosis, prognosis and rehabilitation treatment, the Italian Society of Muscles, Ligaments and Tendons (I.S.Mu.L.T.) proposed a new classification of muscle injuries in 2014 (1). According to the mechanism of injury, muscle injuries are distinguished in to two distinct categories: direct and indirect injuries. A direct injury is defined as involving an external force applied to the muscle and the injury therefore depends on the impact intensity, the state of contraction of the muscle, the traumatic moment and the injured muscle. For indirect injuries, typical of non-contact sports, the main cause of injury is due to internal eccentric muscular force generation, with the main injury site being the muscle tendon junction (MTJ) (20). The structural damage to the muscle fibres may be caused by a singular contraction or by the cumulative effect of multiple contractions (21).

A correct therapeutic approach is crucial to avoid complications and to prevent re-injury. Early complications include compartment syndrome, phlebitis and deep vein thrombosis. Late complications include fibrosis, intermuscular effusion, intramuscular cyst, calcification, intramuscular ossification and muscle hernia (22).

Most muscle injuries are managed conservatively with excellent results, with surgery advocated only for much larger tears. Conservative approaches include many different treatment modalities including POLICE (Protection, Optimal Loading, Ice, Compression, Elevation) protocols, stretching, functional rehabilitation and physical therapies, although there is no consensus on gold-standard management $(23,24)$. A pharmacological and nutritional approach is fundamental to control pain and to promote optimal healing by supporting and optimizing rehabilitation treatments and physiotherapy $(25,26)$. Contrary to the evidence (27), injection treatments for muscle injuries (e.g. platelet-rich plasma) have recently become popular, particularly in professional athletes, with the view to accelerating RTP times. The main indications for surgery are complete lesions and subtotal lesions associated with persistent pain and loss of strength after conservative management (28).

Whichever therapeutic approach is utilised, there should be a strong focus on incorporating re-injury prevention practices, considering all injury risk factors and their interactions, 
through the use of a holistic approach to reduce the risk of re-injury. This should be for both the injured muscle, as well for reducing the risk of other musculoskeletal injuries (29). Prevention programs should be based on multimodal exercise protocols including eccentric training, high speed running, core stability, endurance, flexibility, proprioception, agility and coordination $(30,31)$. Moreover, a specific warm-up before a training session or match can significantly reduce the risk of injury (32). The study was conducted according to the journal's standards (33).

\section{Rationale for an update of the I.S.MU.L.T. classification of muscle injuries}

The I.S.Mu.L.T. classification and guidelines for muscle injuries have provided the scientific world very useful indications and suggestions on epidemiology, diagnosis, classification and management of muscle injuries (1). However, recent reviews of the literature has highlighted the need for an update of this classification system, with a particular focus on three key area.

Firstly, there is a need to focus more precisely on the injury location, distinguishing between proximal, middle and distal sites of the muscle. Indeed, research has highlighted that muscle injuries which occur in the proximal area of the hamstring or rectus femoris muscles are associated poorer prognosis, whilst injury of the triceps surae muscle to the distal regions is associated with longer recovery time (34).

Secondly, it is important to distinguish between the damage tissue type. In particular, ultrasound and MRI can aid in distinguishing between myofascial, muscle fibers, MTJ, and tendon involvement (35).

Thirdly, recent research reports that distinguishing between primary injury and a re-injury is crucial for prognosis and recovery time, and can therefore influence the therapeutic approach $(36,37)$. Muscle re-injury rates are between $12-43 \%$ across different sports. The recovery and RTP times after a re-injury are typically longer than for a primary injury (38). Non-modifiable risk factors for muscle re-injury are age (especially for triceps surae and hamstring) and number of previous injuries of the same or other muscles. Modifiable risk factors include muscle strength deficits, reduced flexibility and muscle fatigue (39). Such modifiable risk factors are directly related to the pathological modifications that usually occurs after an injury, such as stiffness, muscle atrophy, scar tissue formation, biomechanical alterations and neuromuscular control deficits (40). They can also be due to an inadequate rehabilitation management, characterized by an overly aggressive rehabilitation process, incomplete rehabilitation, an underestimation of the previous injury, an overly long immobilization process or an incorrect treatment approach (41).

\section{Updated classification}

\section{Classification}

As we know, muscle injuries are divided in two main categories according to injury mechanism: direct and indirect injuries.

\section{Direct injuries}

Direct muscle injuries are divided into: lacerations and contusion. Lacerations are caused by blunt objects that cause cuts, whilst contusions are caused by direct impact not causing cuts.

Contusions are classified as minor, moderate and severe depending on identified functional deficits assessed by the capacity to engage the muscle and execute its specific action. It is important to re-assess the athlete 24 hours after the injury, as the blunt trauma can be initially overestimated due to the algic component, which limits early movement (1).

\section{Indirect injuries}

Indirect muscle injuries occur without any contact with other players or objects outside the field of play. The athlete essentially injures them self and these types of injury can be divided in two categories:

- non-structural injuries: no anatomic damage or tear, divided into 4 sub-categories;

- structural injuries: characterized by anatomical damage and classified into 3 sub-categories (13).

\section{Non-structural muscle injuries}

Non-structural muscle injuries are the most common injury type, but the diagnosis and treatment can be insidious. In football, they constitute $70 \%$ of all muscle injuries and although they do not involve structural muscle damage, they are still responsible for more than $50 \%$ of muscle injury burden from sports. If neglected they can lead to subsequent structural injuries.

\section{Four non-structural muscle injury subcategories}

- Sub-group 1A is fatigue induced muscle disorder, typical of repetitive movements, inconstant playing surface or by excessive workload volumes.

- Subgroup $1 \mathrm{~B}$ is caused by exercise induced muscle damage resulting in delayed onset of muscle soreness (DOMS).

- Subgroup 2A is caused by spinal problems, often difficult to diagnose, such as minor intervertebral defects, which irritate the corresponding spinal nerve causing altered muscle tone of the respective muscle. In these cases, resolution of the muscle injury requires treating the spinal problem. 
- Subgroup 2B arises from an imbalance in neuromuscular control especially altered reciprocal muscle inhibition. Sensory information from the muscle is carried by ascending pathways to the brain. The afferent signals enter the spinal cord with the alpha motoneurons of the associated muscle, but also give branches capable of stimulating the interneurons in the spinal cord that act in an inhibitory way on the alpha motoneurons of the antagonist muscles. Thereby simultaneous inhibition of the alpha motor neurons to antagonistic muscles (reciprocal inhibition) occurs to support muscle contraction of agonistic muscle. A dysfunction of these neuromuscular control mechanisms can lead to significant impairment of normal muscle tone and can result in neuromuscular disorders, when the inhibition of antagonistic muscles is impaired (e.g. decreased) and the agonist must over-contract to compensate (13).

\section{Structural muscles injuries}

Structural muscle injuries are divided into 3 sub-categories accordingly the severity of the anatomical damage:

- 3A. Partial minor tear - a tear of the primary muscle fascicles inside a secondary muscle (1).

- 3B. Partial moderate tear - a tear of at least one secondary muscle fascicle with $50 \%$ structural damage of the investigated muscle cross sectional area.

- 4. Sub-total tear or complete tear - a tear of at least $50 \%$ structural damage of the investigated muscle cross sectional area. Otherwise complete tear of whole muscle or MTJ.

In addition, considering the I.S.Mu.L.T. classification of 2014, it seems necessary to consider the location of the muscle injury according to its site: proximal, middle, distal.

The classification of muscle structural injuries including the injured location along the muscle:

Proximal site (P); Middle site (M); Distal site (D).

Ultrasound and particularly MRI scans can help to identify involvement of different damaged tissues:

- MF: Myofascial (muscle fibers and fascia);

- MT: Muscle fibers or MTJ;

- T: Tendon.

Myofascial injuries have a more benign prognosis than MTJ and tendon injuries. This for several reasons including i) from the biomechanical point of view, myofascial injuries undergo less traction and stress than the MTJ and tendons and ii) anatomically, the fascia is richly vascularized and therefore has the possibility of "restitutio ad integrum" (restoration to original condition) or better, after a muscle injury.
Anatomically, the MTJ is the muscle portion in which the tendon fibers intertwine until it merges with the muscle fibers. It is the "locus minor resistentiae" (location of least resistance) following specific and eccentric sports movements, which subject the muscle to biomechanical stress. As such, the MTJ is a frequent site of muscle injuries. Physiologically, this is in part due to a histological feature of the myotendinous tissue, which is rich in fast type II fibers, which utilise predominantly anaerobic metabolism. This is important for injury diagnosis and relevant for prognosis, as injuries at the MTJ have longer recovery times than injuries within the muscle belly. Furthermore, injuries with tendon involvement typically have the worst prognosis and may require surgical repair (19). Therefore, it is useful to classify the site of injury, as it is an important prognostic factor.

As described previously, muscle injuries which occur in the proximal area of hamstrings muscle or rectus femoris muscle have longer prognosis compared with muscles injuries at the middle of the muscle, despite similar structural damage. Furthermore, the triceps surae muscles has longer prognosis if the tear is located at the distal level.

A further consideration for these developed guidelines, was the patient's anamnestic history regarding previous injuries. It is important to understand if the injury is a primary injury or first, second or third re-injury (36).

A further innovation of the guidelines is the consideration of injury occurrences:

- Primary injury (R0);

- First re-injury (R1);

- Second re-injury (R2);

- Third re-injury (R3).

We believe recording of the injury as either primary or as a re-injury and the number of re-injuries is essential, as muscle re-injuries are associated with worse prognosis and possibly emphasis a previously failed functional recovery process.

A muscle re-injury is another new injury from non-contact mechanisms in the same location of the previous injury or in the same muscle, occurring within 2 months after RTP (37). RTP times for muscle re-injuries are typically longer than for primary injuries, Recent epidemiological studies on elite football players report muscle re-injury rates of $13-16 \%$ for structural muscle injuries and $12 \%$ for non-structural injuries. Muscle re-injury rates vary between $12-43 \%$ across different sports, including soccer, Australian football, athletics and rugby and result in delayed RTP times. Again, risk factors for muscle injuries can be categorised into modifiable and non-modifiable factors (38).

A few authors reported that muscle re-injuries occur due to multiple co-existing injury risk factors. Deficits in muscle flexibility are considered to associated with muscle re-in- 
Table I. New classification system based on the extent of the tear, tear location, tissue involvement and injury number.

\begin{tabular}{llll}
\hline Tear extent & Tear location & Tissue involved & Re-injury \\
\hline 3A: Partial minor tear & P: Proximal & MF: Myofascial & R0: Primary injury \\
3B: Partial moderate tear & M: Middle & MT: Muscle fibers & R1: First re-injury \\
4: Subtotal or complete tear & D: Distal & and myotendon & R2: Second re-injury \\
& & T: tendon & R3: Third re-injury \\
\hline
\end{tabular}

juries, but not primary muscle injury. Eccentric hamstring strength deficits are associated with higher risk of muscle re-injury. According to the literature, hamstring muscle re-injuries are commonly grade $3 \mathrm{~A}$ and 4 , thought due to miss-diagnosis and failure to identify the severity of the initial injury. Medial gastrocnemius, hamstring and rectus femoris muscles are most susceptible to re-injury (39). The symptoms and clinical signs of the re-injury are similar to the primary injury, so the same considerations of imaging and conservative treatment indications are needed. Muscle re-injury practices should consider the risk factors which may have predisposed the athlete to the initial muscle injury, correct diagnosis of the injury, reduced immobilization times, correct exercises selection, a full and complete functional recovery process as well as management of the athlete after RTP (40). Rehabilitation and/or re-injury prevention programs should include eccentric strengthening at longer muscle lengths, as evidence suggests a protective effect for re-injury upon RTP. Half of muscle re-injured occur during the first month after RTP, thought due to incomplete functional recovery (41).

This new proposed classification considers the magnitude of the tear, localisation of the tear, involved tissue and numbers of re-injury can be seen in table $\mathbf{I}$.

\section{REFERENCES}

1. Maffulli N, Oliva F, Frizziero A, et al. I.S.Mu.L.T. Guidelines for muscle injuries. MLTJ 2014;3(4):241-249.

2. Edouard P, Branco P, Alonso JM. Muscle injury is the principal injury type and hamstring muscle injury is the first injury diagnosis during top-level international athletics championships between 2007 and 2015. Br J Sports Med 2016;50:619-630.

3. Chan O, Del Buono A, Best TM, Maffulli N. Acute muscle strain injuries: a proposed new classification system. Knee Surg Sports Traumatol Arthrosc 2012;20:2356-2362.

4. Ekstrand J, Hägglund M, Waldén M. Epidemiology of muscle injuries in professional football (soccer). Am J Sports Med 2011;39:1226-1232.

5. Bittencourt NFN, Meeuwisse WH, Mendonça LD, et al. Complex systems approach for sports injuries: moving from risk factor identification to injury pattern recognition - narrative review and new concept. Br J Sports Med 2016;50:1309-1314.
Clinical examples using this update classification system:

- long head biceps femoris 3B, P, T, R2: second re-injury with partial moderate tear at proximal site with tendon tissue involvement;

- adductor longus 4, P, MT, R0: primary injury with subtotal or complete tear of the proximal myotendinous junction;

- recuts femoris 3A, M, MF, R1: first re-injury with partial minor tear at middle site with myofascial involvement.

\section{CONCLUSIONS}

To conclude, we believe the proposed classification system is complete, considering the anamnestic, clinical and localization aspects of muscle injuries, which should support clinicians in determining muscle injury prognosis. Such an integrated classification can support the scientific community to have consistent methods of reporting muscle injuries. This could aid in the development of more targeted and evidencebased treatments to support athletes to RTP early and safely, as well as minimizing the risk for muscle re-injury.

\section{CONFLICT OF INTERESTS}

The authors declare that they have no conflict of interests.

6. Maffey L, Emery CA. What are the risk factors for groin strain injury in sport? A systematic review of the literature. Sports Med 2007;37:881-894.

7. Keller K, Engelhardt M. Strength and muscle mass loss with aging process. Age and strength loss. MLTJ 2014;24;3:346-350.

8. Hägglund M, Waldén M, Magnusson $H$, et al. Injuries affect team performance negatively in professional football: an 11-year follow-up of the UEFA Champions League injury study. Br J Sports Med 2013;47:738-742.

9. Van Dyk N, Bahr R, Whitley R, et al. Hamstring and quadriceps isokinetic strength deficits are weak risk factors for hamstring strain injuries: A 4-year cohort study. Am J Sports Med 2016;44(7):1789-1795.

10. Ivarsson A, Johnson U, Andersen MB, et al. Psychosocial factors and sport injuries: Meta-analyses for Prediction and Prevention. Sports Med 2017;47:353-365. 
11. Bengtsson H, Ekstrand J, Waldén M, et al. Muscle injury rate in professional football is higher in matches played within 5 days since the previous match: a 14-year prospective study with more than 130000 match observations. Br J Sports Med 2018;52(17):1116-1122.

12. Pfirrmann D, Herbst $M$, Ingelfinger $P$, et al. Analysis of Injury Incidences in Male Professional Adult and Elite Youth Soccer Players: A Systematic Review. J Athl Train 2016;51(5):410-424.

13. Mueller-Wohlfahrt HW, Haensel L, Mithoefer K, et al. Terminology and classification of muscle injuries in sport: the Munich consensus statement. Br J Sports Med 2013;47(6):342-350.

14. Kary JM. Diagnosis and management of quadriceps strains and contusions. Curr Rev Musculoskelet Med 2010;3(1-4):26-31.

15. Manara M, Manari D, Pasta G. Diagnostic Imaging in Muscle Injury. In: Muscle Injuries in Sport Medicine. InTech 2013;3:97-134.

16. Crema M, Yamada A, Guermazi A, et al. Imaging techniques for muscle injury in sports medicine and clinical rele- vance. Curr Rev Musculoskelet Med 2015;8(2):154-161.

17. Ekstrand J, Lee J, Healy J. MRI fin- dings and return to play in football: a prospective analysis of 255 ham- string injuries in the UEFA Elite Club Injury Study. Br J Sports Med 2016;50(12):738-743.

18. Ekstrand J, Askling C, Magnusson H, et al. Return to play after thigh muscle injury in elite football players: implementation and validation of the Munich muscle injury classification $\mathrm{Br} \mathrm{J}$ Sports Med 2013;00:1-6.

19. Pollock N, Patel A, Chackraverty J, et al. Time to return to full training is delayed and recurrence rate is higher in intratendinous ('c') acute hamstring injury in elite track and field athletes: clinical application of the British Athletics Muscle Injury Classification. Br J Sports Med 2016;50:305-310.

20. Malliaropoulos NG. Non contact Hamstring injuries in sports. MLTJ 2013;2:309-311.

21. Jarvinen TA, Jarvinen TL, Kaariainen M, et al. Muscle injuries: biology and treatment. Am J Sports Med 2005;33:745-764.

22. Alessandrino F, Balconi G. Complications of muscle injuries. J Ultrasound 2013;16:215-222.

23. Maffulli N, Del Buono A, Oliva F, et al. Muscle Injuries: A Brief Guide to Classification and Management. Transl Med UniSa 2014;12:14-1218.

24. Bleakley C, Glasgow P, MacAuley D. PRICE needs updating, should we call the POLICE? Br J Sports Med 2012;46: 220-221.

25. Mackey AL, Mikkelsen UR, Magnusson SP, et al. Rehabilitation of muscle after injury - the role of anti-inflammatory drugs. Scand J Med Sci Sports 2012;22(4):e8-14.

26. Morton RW, Murphy KT, McKellar SR, et al. A systematic review, meta-analysis and meta-regression of the effect of protein supplementation on resistance training-induced gains in muscle mass and strength in healthy adults. Br J Sports Med 2018;52(6):376-384.
27. Hamid MSA, Mohamed Ali MR, Yusof A, et al. Platelet-rich plasma injections for the treatment of hamstring injuries: a randomized controlled trial. Am J Sport Med 2014;42(10):2410-2418.

28. Oliva F, Via AG, Kiritsi O, Foti C, Maffulli N. Surgical repair of muscle laceration: biomechanical properties at 6 years follow-up. MLTJ 2014;3:313-317.

29. Brukner P, Nealon A, Morgan C, et al. Recurrent hamstring injury: applying the limited evidence in the professional football setting with a seven-point programme. Br J Sports Med 2014;48:929-938.

30. Al Attar WS, Soomro N, Sinclair PJ, et al. Effect of injury prevention programs that include the Nordic hamstring exercise on hamstring injury rates in soccer players: a systematic review and meta-analysis. Sports Med 2017;47(5):907-916.

31. Lauersen JB, Bertelsen DM, Andersen LB. The effectiveness of exercise interventions to prevent sports injuries: a systematic review and meta-analysis of randomised controlled trials. $\mathrm{Br} \mathrm{J}$ Sports Med 2014;48:871-877

32. Sadigursky D, Braid JA, De Lira DNL, et al. The FIFA 11+ injury prevention program for soccer players: a systematic review. BMC Sports Sci Med Rehabil 2017;9:18

33. Padulo J, Oliva F, Frizziero A, Maffulli N. Muscles, Ligaments and Tendons Journal - Basic principles and recommendations in clinical and field Science Research: 2018 update. MLTJ 2018;8(3):305-307.

34. Hamilton B, Valle X, Rodas G, et al. Classification and grading of muscle injuries: a narrative review. $\mathrm{Br} \mathrm{J}$ Sports Med 2015;49(5):306

35. Pollock N, Patel A, Chackraverty J, et al. Time to return to full training is delayed and recurrence rate is higher in intratendinous ('c') acute hamstring injury in elite track and field athletes: clinical application of the British Athletics Muscle Injury Classification. Br J Sports Med 2016;50:305-310.

36. Valle X, Alentorn-Geli E, Tol JL, et al. Muscle Injury in Sports: A New Evidence-Informed and Expert Consensus-Based Classification and Clinical Application. Sports Med Auckl NZ 2017;47:1241-1253.

37. Järvinen TA, Järvinen M, Kalimo H. Regeneration of injured skeletal muscle after the injury. MLTJ 2014;24;3(4):337-345.

38. Carling C, Le Gall F, Orhant E. A four-season prospective study of muscle strain reoccurrences in a professional football club. Res Sports Med 2011;19(2):92-102.

39. Hagglund M, Waldén M, Ekstrand J. Risk factors for lower extremity muscle injury in professional soccer: the UEFA Injury Study. Am J Sports Med 2013;41(2):327-335.

40. Fyfe JJ, Opar DA, Williams MD, et al. The role of neuromuscular inhibition in hamstring strain injury recurrence. J Electromyogr Kinesiol 2013;23(3):523-530.

41. Opar DA, Williams MD, Shield AJ. Hamstring strain injuries: factors that lead to injury and re-injury. Sports Med 2012;42(3):209-226. 